\title{
Analysis of HD 73045 light curve data
}

\author{
Mrinal Kanti Das ${ }^{1 *}$, Naveen Kumar Bhatraju ${ }^{2}$, Santosh Joshi ${ }^{3}$
}

${ }^{1}$ Institute of Informatics and Communication, University of Delhi South Campus, New Delhi, India

${ }^{2}$ CSIR-Institute of Genomics and Integrative Biology, Mall Road, Delhi 110007, India

${ }^{3}$ Aryabhatta Research Institute of Observational Sciences (ARIES), Nainital 263002, India

\begin{abstract}
In this work we analyzed the Kepler light curve data of HD 73045. The raw data has been smoothened using standard filters. The power spectrum has been obtained by using a fast Fourier transform routine. It shows the presence of more than one period. In order to take care of any non-stationary behavior, we carried out a wavelet analysis to obtain the wavelet power spectrum. In addition, to identify the scale invariant structure, the data has been analyzed using a multifractal detrended fluctuation analysis. Further to characterize the diversity of embedded patterns in the HD 73045 flux time series, we computed various entropy-based complexity measures e.g. sample entropy, spectral entropy and permutation entropy. The presence of periodic structure in the time series was further analyzed using the visibility network and horizontal visibility network model of the time series. The degree distributions in the two network models confirm such structures.
\end{abstract}

\section{Introduction}

In this paper, we study the time series obtained with the Kepler space mission of the star HD 73045 in the linear and nonlinear domain. In the linear domain, assuming the process of generating the time series to be stationary, a conventional Fourier transform of the time series of HD 73045 flux has been carried out. In order to investigate the temporal structure and possible nonstationary behavior of the time series, a continuous wavelet transform has been obtained (Torrence \& Compo 1998). In any dynamical system (e.g. simple stellar pulsation models; Bhatraju \& Das 2014), the temporal dynamics of dynamical variables may exhibit nonlinear characteristics (like a cascade of periodic doubling chaos, multi-fractality etc.) depending on the parameters controlling the dynamics. Several authors (Kiss \& Szatmary 2002, and references therein) invoked the nonlinear framework to interpret the observed irregularity in a large number of light curves of W Virginis, R Cygni, AC Herculis, etc. Further complex systems are low dimensional chaotic systems and are likely to exhibit multifractal behavior (cf. Kantelhardt et al. 2002). To unravel the embedded scale invariant characteristics in the HD 73045 flux time series, a multifractal detrended fluctuation analysis (MFDFA) is applied (cf. Das et al. 2012). The observed decreasing trend of the generalized Hurst exponent $(h(q))$ and the multifractal singularity spectrum clearly suggest the multifractal behavior of the HD 73045 time series. In this paper, to further characterize the diversity of patterns in the HD 73045 data, we have also obtained

\footnotetext{
*e-mail: dasmkd11@gmail.com
} 


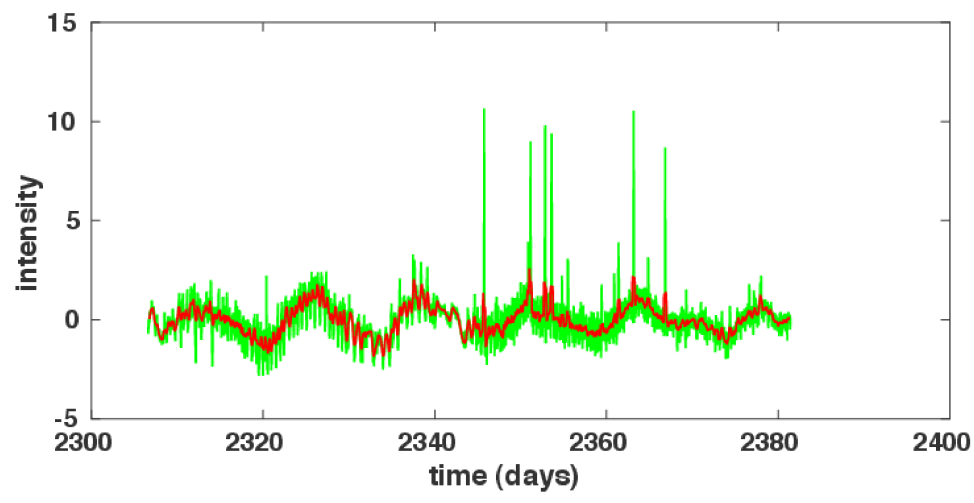

Figure 1: Time series of HD 73045 obtained with the Kepler satellite mission. Raw and smoothed data are shown in green and red, respectively. The time shown on the X-axis corresponds to BJD-2 454833.

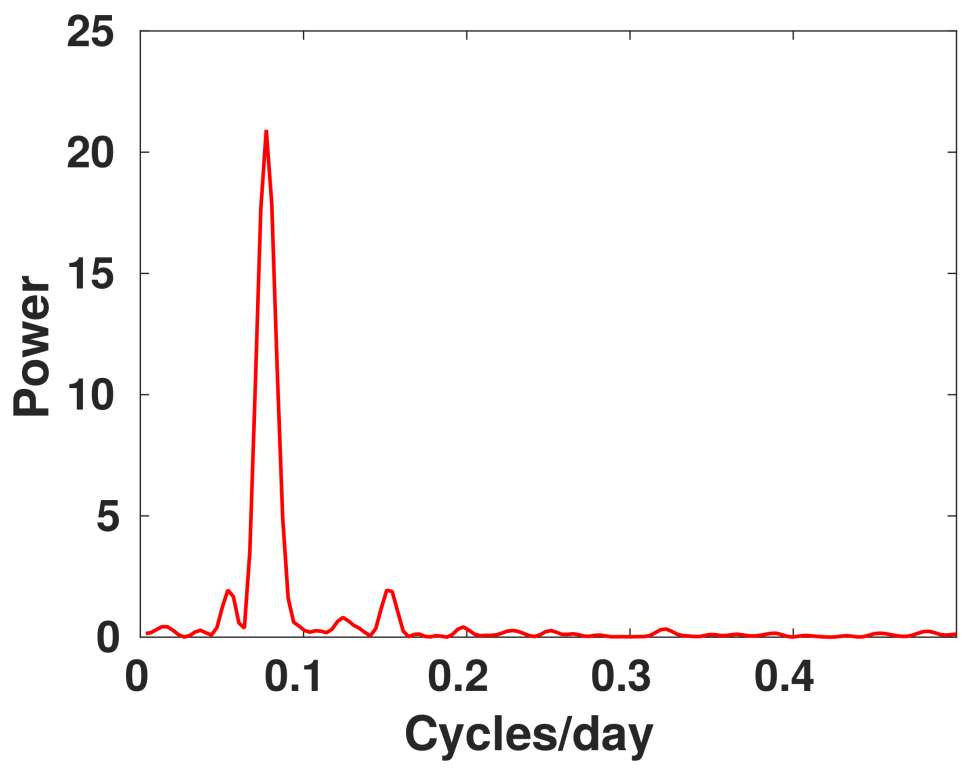

Figure 2: Fast Fourier transform (FFT) of the time series of HD 73045 shown in Fig. 1.

values of various entropy-based complexity measures like the spectral entropy $\left(E_{m}\right)$, the sample entropy (SampEn), and the permutation entropy (PE) (Semmlow \& Griffel 2014). Following Xie \& Zhou (2011), the Lacasa \& Toral (2010) network models of the time series of HD 73045 have been constructed to characterize it as either regular or chaotic, using the visibility graph and horizontal visibility graph model.

\section{Wavelet and Fourier analysis}

The time series of HD 73045 observed by the Kepler space mission (shown in Fig. 1) can be considered as a smooth signal $x_{n}$, with $n=1,2, \ldots, N$ (= the number of observations). It was used to calculate the Fast Fourier Transform (FFT; Lomb 1976; see Fig. 2) and the continuous wavelet transform (CWT; Torrence \& Compo 1998; see Fig. 3). Both the FFT and CWT results in the presence of two periods of $\sim 13.1$ and $\sim 6.4$ days, respectively. It is interesting to note the presence of the subharmonic in HD 73045 data, which also suggests its nonlinear behavior. 


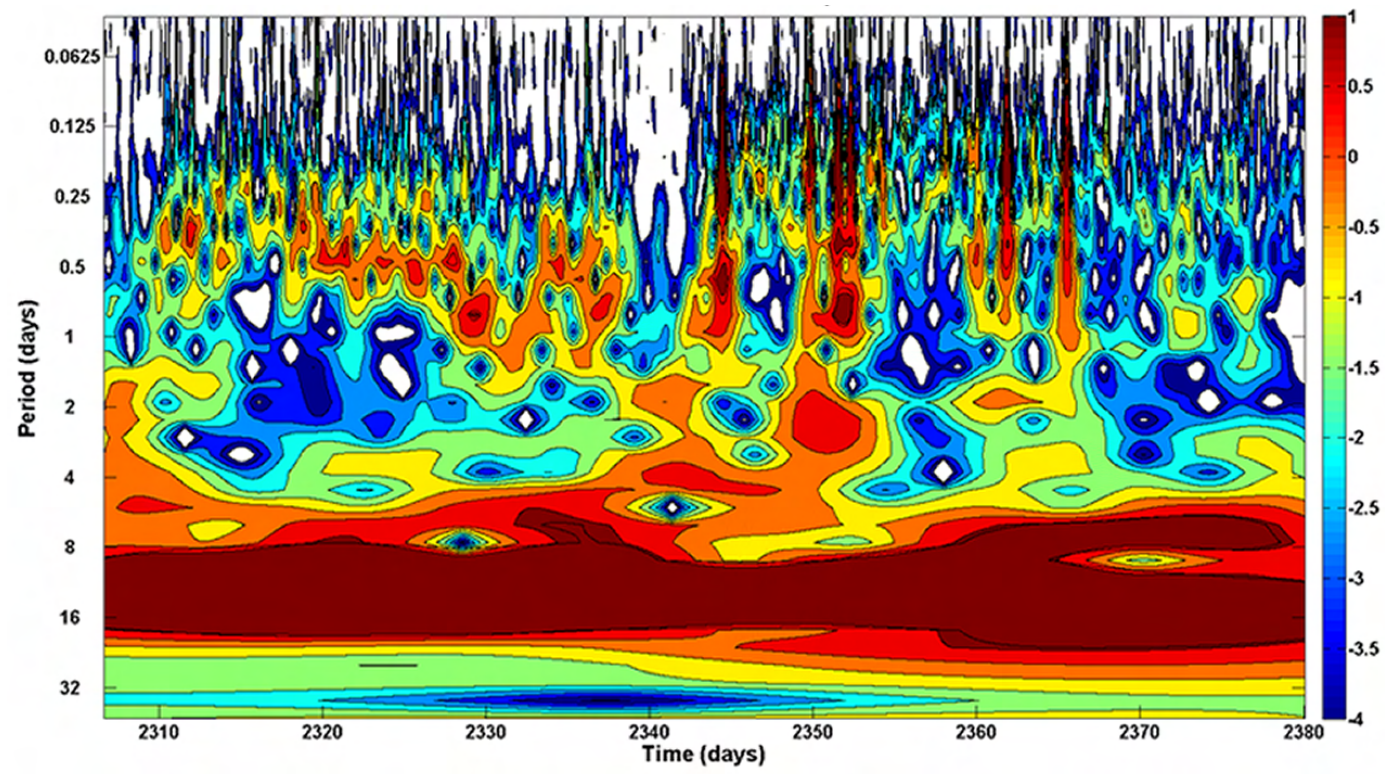

Figure 3: Continuous wavelet transform (CWT) of the time series of HD 73045 shown in Fig. 1.

\section{Multifractal behavior}

For the application of MFDFA, following Kantelhardt et al. (2002), we define a profile $Y(i)=$ $\sum_{n=1}^{i}\left(x_{n}-<x>\right)$ for $i=1, \ldots, N$, and we divide it into $N_{s} \equiv \operatorname{int}(N / s)$ non-overlapping segments of equal length $s$. Here $\langle x\rangle$ refers to mean of $x$. The local variance for each segment $\nu$, with $\nu=1, \ldots, N_{s}$, is given by:

$$
F^{2}(\nu, s)=\frac{1}{s} \sum_{i=1}^{s}\left\{Y[i+(\nu-1) s]-Y_{\nu}(i)\right\}^{2}
$$

where $Y_{\nu}(i)$ is the least square quadratic fit to each of the $N_{s}$ segments. Using the local variance $F^{2}(\nu, s)$, we obtain the expression for generalized fluctuations in terms of generalized Hurst exponents $h(q)$, as:

$$
F_{q}(s)=\left\{\frac{1}{N_{s}} \sum_{\nu=1}^{N_{s}}\left[F^{2}(\nu, s)^{q / 2}\right]\right\}^{1 / q} \sim s^{h(q)}
$$

where, $h(q)$ is the generalized Hurst exponent (see Di Matteo et al. 2003). For a monofractal, $h(q)$ is a constant while for multifractals it varies with $q$. It is also observed that the scaling index $\tau(q)$ and the singularity spectrum $f(\alpha)$, where $\alpha$ is the singularity strength (also known as the Hölder exponent), are related to the Hurst exponents as: $\tau(q)=q h(q)-1 ; \alpha=\tau^{\prime}(q)$ and $f(\alpha)=q \alpha-\tau(q)$. It is to be noted that $f(\alpha)$ encodes all global scaling information. Various plots in Fig. 4 (left and right) suggests multifractal behavior of the time series for HD 73045. Multifractal analysis sheds light on the scale invariant structure existing in the light flux data. Further, the parameter $h(q=$ 2) (correlation dimension) quantifies the long-term correlations present in the data. Over all, the observed multifractal behaviour may have implications for the determination of the intermittency inherent to the HD 73045 time series.

\section{Complexity measures}

Entropy is important for characterizing the diversity of patterns in a given time series. It is advantageous to use the entropy approach for time series analysis as it takes into account the nonlinear 

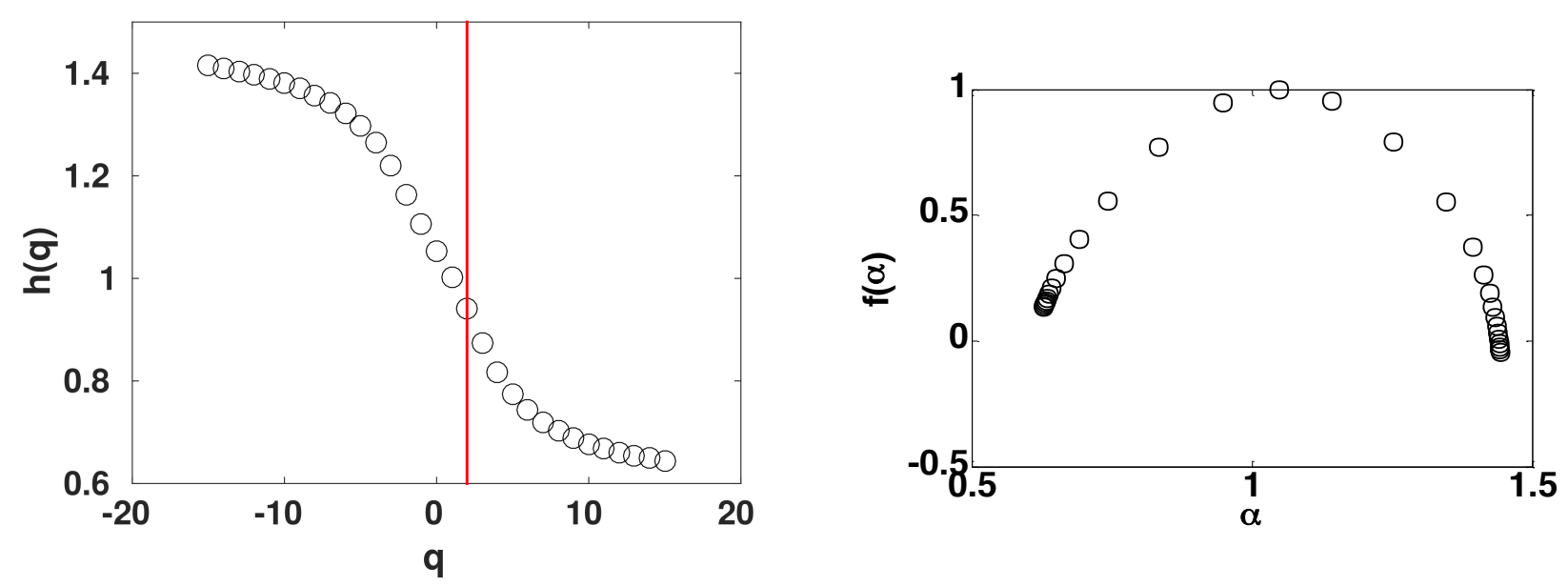

Figure 4: (left) The generalized Hurst exponent $h(q)$ as a function of $q$. The intersection of the red line with the curve marks the value $h(q=2)$. (right) The singularity spectrum $f(\alpha)$ as a function of the singularity strength $\alpha$.

dependencies in the autocorrelation structure of the time series. It is to be noted that entropy provides an index of the quantity of information contained in the data sequence. A high/low value of entropy corresponds to a lower/higher predictability of the involved dynamics. Moreover, it can also be used for the short sequence of Kepler data used in this study. To quantify the complex behavior of the HD 73045 data, we computed the various entropy-based measures $E_{m}, S a m p E n$, and PE (Bandt \& Pompe 2002). For the given time series of HD 73045, the computed values of $E_{m}$, SampEn, and $P E$ are found to be $0.6907,1.664$, and 1.7147 , respectively. While the low value of the spectral entropy relates to the linear structure prevailing in the data, the higher values of both the sample and permutation entropy reflects the presence of nonlinear structures in the time series of HD 73045.

\section{Network model}

Nonlinear time series analysis provides an understanding of the dynamical processes involved in generating a time series. Besides the foregoing techniques, the dynamics of the system can also be partially revealed by analyzing the corresponding network's topological properties. There are several methods available to construct a network of a given time series. Here we consider the visibility graph (VG) and the horizontal visibility graph (HVG) method to obtain the network model for the HD 73045 data (cf. Lacasa \& Toral 2010).

It is noted that the degree distribution embeds the information concerning the original time series. For instance, a random time series results in a uniform distribution. The HVG is a variant of the VG and it is defined such that it has a Hamiltonian path. For the HD 73045 data, we have constructed VG and HVG networks using the HD 73045 flux data. In a network, the degree $K$ of a vertex corresponds to number of edges connected to it. Therefore, on finding the distribution function $P(K)$ for degree $K$ (i.e. the relative frequency associated with degree $K$ ), we obtain Fig. 5 showing the degree distribution. It is interesting to find that the majority of the points are aligned along a straight line. It suggests a noisy periodic behavior of the time series, as has also been shown by Lacasa \& Toral (2010). 

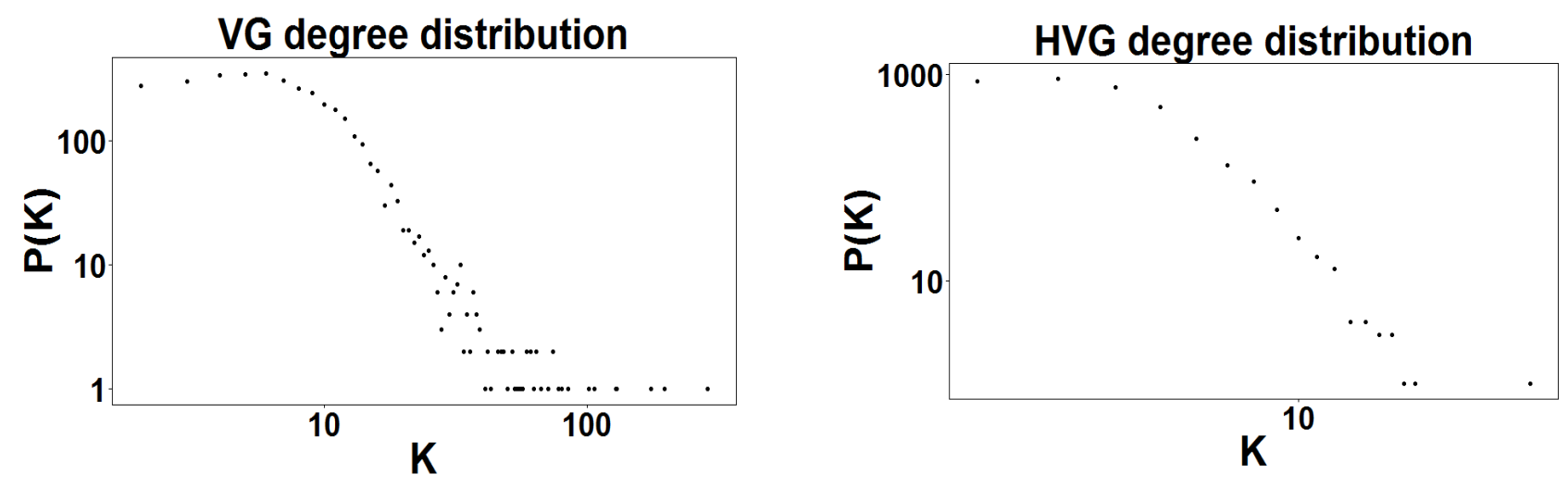

Figure 5: The VG degree distribution (left) and HVG degree distribution (right) $P(K)$ as a function of the degree $K$.

\section{Results}

In our nonlinear analysis of the time series of HD 73045 as observed by the Kepler mission based on a FFT and wavelet analysis, we showed that the time series are dominated by two periodic signals with periods of 13.1 and 6.4 days, respectively. However, a very large number of transient noisy lowamplitude periods are prevalent in the data as well. The MFDFA analysis of the data clearly points to multifractal behavior, thereby suggesting the presence of many scale invariant operating structures. It is significant to observe the long term correlation in the data as indicated by the correlation dimension value of $\sim 0.95$, i.e. the value of the generalized Hurst exponent $h(q)$ for $q=2$. The nonlinear variability of the HD 73045 data is characterized by the entropy-based complexity indices as given by the spectrum entropy $E_{m}=0.6907$, the sample entropy $S a m p E n=1.664$, and the permutation entropy $P E=1.7147$, respectively. Recently, network models have been introduced as an alternative framework for the analysis of time series. In the present work, we computed the VG and HVG network models for the data of HD 73045. The degree distribution of various nodes in both the VG and its variant $\mathrm{HVG}$ shows the presence of periodic structures embedded in noise. Besides providing insight into the linear, non-linear, fractal and periodic structures in the time series, these parameters were found to be useful in building novel classifiers in various scientific areas such as medicine, Earth sciences, atmospheric sciences, etc. In this regard, we propose to extend the present variable star classification framework to incorporate the results of non-linear analyses like the ones we used in this work.

\section{References}

Bandt C., Pompe B. 2002, PhRvL, 88, 174102.

Bhatraju Naveen K., Das M. K. 2014, Ap\&SS, 352, 83

Das M. K., Bhattacharjee A., Bhatraju Naveen K., Yuasa M., Saha L. M. 2012, Bulletin of Calcutta Mathematical Society, 104, 561

Di Matteo T., Aste T., Dacorogna M. M. 2003, Physica A, 324, 183

Kantelhardt J. W., Zschiegner S. A., Koscielny-Bunde E. et al. 2002, PhyA, 316, 87

Kiss L. L., Szatmary K. 2002, A\&A, 390, 585

Lacasa L., Toral R. 2010, Phys.Rev. E., 82, 036120

Lomb N. R. 1976, Ap\&SS, 39, 447

Semmlow J., Griffel B. 2014, Biosignal and medical image processing, CRC Press

Torrence C., Compo G. 1998, BAMS, 79, 61

Xie W., Zhou W. 2011, PhyA, 390, 3592 